\title{
Diffusion and ion-exchange properties for conservative and non- conservative ions in granite: An X- ray radiography method
}

\author{
Charles CadieuX ${ }^{1}$, SAMuel Morfin ${ }^{1}$, Guadalupe \\ MALDONADO $^{1}$, TOM AL ${ }^{1, *}$ \\ ${ }^{1}$ Department of Earth and Environmental Sciences, \\ University of Ottawa, Ottawa, ON, K1N 6N5, Canada \\ [*correspondence: tom.al@uottawa.ca]
}

Deep geological repositories for radioactive waste disposal are key for the long-term viability of nuclear energy. Granites are of interest because they provide a low permeability barrier against solute migration via diffusion. They also provide sorption capacity, although the in-situ sorption capacity of granitic rocks is not well characterized. X-ray radiography has been used to measure diffusion coefficients and sorption properties [1,2], but the low porosity and heterogeneity of granites pose challenges. Here we present modifications that allow measurement of diffusion and sorption properties of granites with $<0.5 \%$ porosity.

The radiography method is based on X-ray absorption by high-atomic-number aqueous tracers as they diffuse through a porous media. Image contrast from the $\mathrm{X}$-ray absorption allows for estimation of diffusion coefficients and measurement of porosity. Compared to more conventional methods, such as through-diffusion, the technique is rapid, non-destructive, and provides time- and spatially-resolved data. However, in low porosity rocks, the signal from the tracers is difficult to detect and the signal-to-noise ratio is prohibitively low. Furthermore, in experiments with medium- to coarse-grained heterogenous granites, image registration errors are large relative to the measured signal. Here we report on an approach that largely eliminates error associated with registration by rotating the sample continuously during image acquisition. With this method it is possible to detect diffusion profiles for conservative $\left(2 \mathrm{M} \mathrm{I}^{-}\right)$and reactive $\left(1 \mathrm{M} \mathrm{Cs}^{+}\right)$tracers in granite.

Fitting this data to an analytical solution of Fick's second law will allow estimation of pore diffusion coefficients [1] for I. Reactive-transport modeling coupled with parameter estimation software will be used to obtain pore diffusion coefficients, selectivity coefficients and cation exchange capacity (CEC) for $\mathrm{Cs}^{+}$[2]. We will report on the results of these analyses.

[1] Cavé et al. (2009) J. Contam. Hydrol. 10(1-2), 1-12.

[2] Loomer et al. (2013) Applied Geochemistry 39, 49-52. 